\title{
Predictors of survival in patients with pulmonary hypertension and acute right heart failure
}

\author{
Ambroz $\mathrm{D}^{1}$, Jansa $\mathrm{P}^{1}$, Kuchar $\mathrm{J}^{2}$, Belohlavek $\mathrm{J}^{1}$, Aschermann $\mathrm{M}^{1}$, Dytrych $\mathrm{V}^{1}$, Lindner $\mathrm{J}^{3}$, \\ Simkova $\mathrm{I}^{4}$, Linhart $\mathrm{A}^{1}$ \\ Clinical Department of Cardiology and Angiology, 1st Faculty of Medicine,2nd Medical Department, \\ Charles University, Prague, Czech Republic. pavel.jansa@vfn.cz
}

\begin{abstract}
OBJECTIVES: The aim of this study was to analyse survival of patients with pulmonary arterial hypertension $(\mathrm{PAH})$ and chronic thromboembolic pulmonary hypertension (CTEPH) hospitalized due to an acute right heart failure (ARHF) with emphasis on risk factors and effectiveness of treatment following current guidelines. METHODS: We retrospectively analysed 117 hospitalizations of 70 patients (59 PAH patients; 11 CTEPH patients, mean age $53.1 \pm 16.77$ years, $54 \%$ females) between 2004 and 2013 .

RESULTS: 96 cases were hospitalized at cardiology wards (CW) while 21 at intensive care unit (ICU). The overall hospital mortality was $12.8 \%$, CW mortality was $4 \%$, and ICU mortality was $52.4 \%$. Higher risk of in-hospital mortality was associated with younger age, lower sodium levels, severe forms of PAH (heritable PAH, CTD-PAH) and need of PAH combination treatment. The one-year survival from the first ARHF hospitalization was $67.6 \%$ (95\% Cl 57.1-80 \%), the two-year survival was $41.9 \%$ (95 \% Cl 30.8-56.9\%). The presence of ascites was a predictor of long-term mortality. CONCLUSIONS: Mortality in patients with PH and ARHF remains very high. Identification of its risk factors could be used as basis of risk-adapted therapy (Tab. 5, Fig. 2, Ref. 14). Text in PDF www.elis.sk. KEY WORDS: acute right hear failure, pulmonary arterial hypertension, chronic thromboembolic pulmonary hypertension, mortality.
\end{abstract}

\section{Introduction}

Acute right heart failure (ARHF) is a leading cause of morbidity and mortality in patients with pulmonary arterial hypertension (PAH) and chronic thromboembolic pulmonary hypertension (CTEPH) (1). The latest published data on causes of ARHF in those patients originate from data on patients treated by 2009 (2, 3 ). However, the last decade was characterised by the availability of advanced PAH treatment (both oral and parenteral specific vasodilation treatment, both individually and combined, that demonstrably affects both symptoms and prognosis of the patients $(4,5))$ as well as CTEPH treatment (causal treatment by surgical pulmonary endarterectomy, PEA, with a significant effect on patients' outlook, as well as pulmonary hypertension-specific phar-

${ }^{1}$ Clinical Department of Cardiology and Angiology, 1st Faculty of Medicine,2nd Medical Department, Charles University, Prague, Czech Republic, ${ }^{2}$ Department of Cardiology, Tabor Hospital, Inc., Czech Republic, ${ }^{3}$ Department of Cardiovascular Surgery, 1 st Faculty of Medicine, 2nd Department of Surgery, Charles University, Prague, Czech Republic, and ${ }^{4}$ Slovak Medical University and The National Institute for Cardiovascular Diseases, Bratislava, Slovakia

Address for correspondence: P. Jansa, Clinical Department of Cardiology and Angiology, 1st Faculty of Medicine, 2nd Medical Department, Charles University, U Nemocnice 2, CZ-128 08 Praha 2, Czech Republic. Phone: +420.224962630 , Fax: +420 224912154

Acknowledgements: This work was supported by the grant Progres Q38/LF1. macotherapy in patients with inoperable disease or with residual pulmonary hypertension after surgical treatment). In addition, significant advances in intensive care and heart failure treatment were made $(1,6,7,8)$.

The aim of our retrospective study was to analyse the outcome of patients with PAH and CTEPH treated in accordance with the current guidelines in departments where all of the recommended treatment options were available between 2004 and 2013. The presented study builds on data collected at the centre for Pulmonary Hypertension at the General University Hospital in Prague and at the Department of Cardiology and Angiology, Slovak Medical University and National Institute of Cardiovascular Diseases, Bratislava, Slovakia (Centre for CTEPH).

\section{Patients and methods}

\section{Patient population}

We performed a retrospective analysis of medical records of 117 hospitalizations for ARHF in 70 patients with chronic $\mathrm{PH}$ (59 patients with $\mathrm{PAH}$ and 11 patients with CTEPH, mean age 53.1 \pm 16.77 years; $46 \%$ male). The diagnosis of PAH and CTEPH, hospital care and subsequent follow-up on outpatient basis were performed according to current guidelines. The definite diagnosis was made by right heart catheterization with haemodynamic evidence of precapillary pulmonary hypertension with a pulmonary artery mean pressure (PAMP) $\geq 25 \mathrm{~mm} \mathrm{Hg}$, pulmonary capillary 
wedge pressure $(\mathrm{PCWP}) \leq 15 \mathrm{~mm} \mathrm{Hg}$ and pulmonary vascular resistance $(\mathrm{PVR}) \geq 3$ Wood Units. CTEPH diagnosis was based on the same criteria combined with a corresponding finding by at least two imaging methods (CT angiography, V/Q scintigraphy, pulmonary angiography) (9).

On admission for ARHF, all patients have undergone a complete routine baseline work-up in accordance with contemporary standards including assessment of medical history, concomitant diseases, full laboratory tests and/or other noninvasive methods. Data on the ward of hospitalization (ICU/cardiology ward) and method of treatment (pharmacotherapy, organ support...) were recorded, as well as available data on the triggering factors of the ARHF and on mortality during hospitalization. Surviving patients were regularly followed up at specialized pulmonary hypertension outpatient clinics of both participating hospitals. Data on survival up to 31.12.2014 were recorded and association of the individual parameters with in-hospital and long-term mortality were analysed. We have also focused on the differences between ARHF treatment at ICU and cardiology wards.

\section{Statistical analysis}

Statistical analysis was performed using R-project, version 3.2.3. Results are expressed as mean, median and standard deviation for continuous variables or as the number of cases and percentages for categorical variables. T-test was used for comparison of normally distributed variables and Mann-Whitney test for comparison of abnormally distributed variables. Categorical variables were compared using Fisher's exact test. Logistic regression was used to evaluate in-hospital mortality. Generalized estimating equations were used to account for repeated measurements. Cox proportional hazard model and log-rank test were used to assess the effect of covariates on the survival; $\mathrm{p}<0.05$ was considered statistically significant.

The study was conducted in accordance with the principles laid down in the 18th World Medical Assembly (Helsinki, 1964), including all subsequent amendments, and in compliance with all local laws and regulations. The approval of the study was provided by the institutional ethics committee.

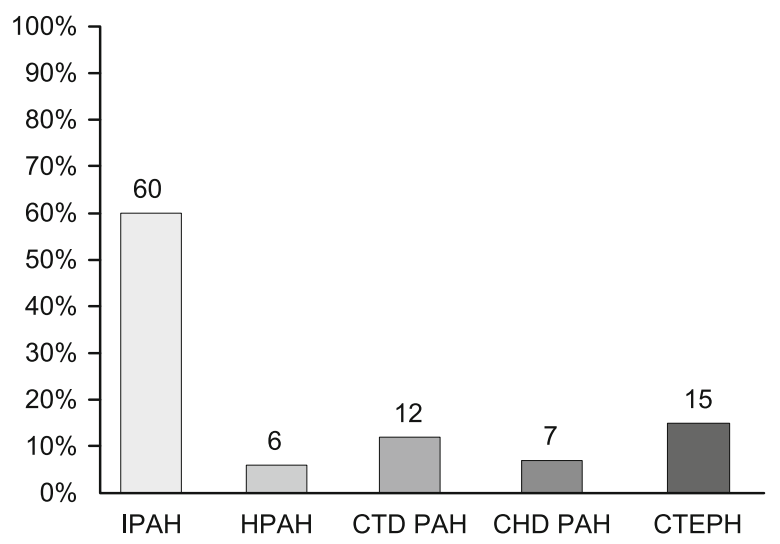

Fig. 1. Aetiology of pulmonary hypertension in patients hospitalized for ARHF.
Tab. 1. Overall baseline characteristics of patients.

\begin{tabular}{|c|c|}
\hline \multicolumn{2}{|l|}{ Population } \\
\hline Subjects (n) & 70 \\
\hline ARHF hospitalizations (n) & 117 \\
\hline Age at first admission (years) & $53.1 \pm 16.77$ \\
\hline Female (n; \%) & $38(54 \%)$ \\
\hline Height (cm) & $168.8 \pm 9.3$ \\
\hline Weight $(\mathrm{kg})$ & $81.1 \pm 20.4$ \\
\hline $\mathrm{BMI}\left(\mathrm{kg} / \mathrm{m}^{2}\right)$ & $28.3 \pm 6$ \\
\hline \multicolumn{2}{|c|}{ Type of pulmonary hypertension (n, \%) } \\
\hline IPAH & $42(60 \%)$ \\
\hline НРAH & $4(6 \%)$ \\
\hline CTD & $8(12 \%)$ \\
\hline CHD & $5(7 \%)$ \\
\hline CTEPH & $11(15 \%)$ \\
\hline \multicolumn{2}{|c|}{ Right heart catheterisation at diagnosis } \\
\hline RA & $12.5 \pm 6.4 \mathrm{mmHg}$ \\
\hline PAMP & $55.1 \pm 14.1 \mathrm{mmHg}$ \\
\hline PCWP & $10.7 \pm 3.1 \mathrm{mmHg}$ \\
\hline $\mathrm{CO}$ & $4.0 \pm 0.91 / \mathrm{min}$ \\
\hline PVR & $11.6 \pm 4.6$ W.U. \\
\hline \multicolumn{2}{|c|}{ Pulmonary hypertension vasodilatory treatment: } \\
\hline without specific therapy & $7(10 \%)$ \\
\hline sildenafil & $39(56 \%)$ \\
\hline endothelin receptor antagonist & $19(27 \%)$ \\
\hline riociguat & $5(7 \%)$ \\
\hline prostanoids & $29(41 \%)$ \\
\hline combined therapy & $28 \quad(40 \%)$ \\
\hline
\end{tabular}

IPAH - idiopathic PAH, HPAH - heritable PAH, CTD - connective tissue disease PAH, CHD - congenital heart disease PAH, CTEPH - chronic thromboembolic pulmonary hypertension, RA - right atrium, PAMP - pulmonary artery mean pressure, PCWP - pulmonary capillary wedge pressure, $\mathrm{CO}$ - cardiac output, $\mathrm{PVR}$ - pulmonary vascular resistance, Continuous parameters are described by mean $\pm \mathrm{SD}$. Categorical parameters are described using absolute (relative) frequency, relative frequencies are only calculated from known data

\section{Results}

\section{Patient characteristics}

In total, 70 patients with 117 admissions for ARHF were included in the study. 21 admissions were hospitalized in ICU (17.9 $\%)$. Hemodynamic instability and/or multiorgan failure were indications for ICU hospitalization. 96 admissions were hospitalized at cardiology wards (72.1\%). 42 patients were hospitalized with a diagnosis of idiopathic PAH ( $60 \%), 4$ patients with heritable PAH (6 $\%$ ), 8 patients with connective tissue disease-associated PAH (CTDPAH $12 \%), 5$ patients with congenital heart disease associated PAH (CHD-PAH $7 \%$ ) and 11 patients with CTEPH (15\%) (Fig. 1). The median duration of a hospital stay was 13.0 days (1-288 days).

Haemodynamic data from all patients at the time of diagnosis along with basic demographic parameters are summarized in Table 1.

Laboratory and clinical presentation on admission for ARHF

Hyponatremia (natrium $\leq 136 \mathrm{mmol} / \mathrm{L}$ ) on admission was present in $63(53.3 \%)$ hospitalizations, severe hyponatremia (with natrium $\leq 130 \mathrm{mmol} / \mathrm{L})$ was recorded in $21(17.9 \%)$ hospitalizations.

On admission, mean creatinine level in patients admitted to $\mathrm{CW}$ was $127.9 \pm 46.91 \mathrm{umol} / \mathrm{L}$; in patients admitted to ICU, the mean value was $153.9 \pm 74.21 \mathrm{umol} / \mathrm{L}$, the difference was not sta- 
Tab. 2. Baseline characteristics of patients at ICU and Cardiology wards.

\begin{tabular}{lccc}
\hline & Total & ICU & $\begin{array}{c}\text { Cardiology } \\
\text { ward }\end{array}$ \\
\hline N of cases & 117 & 21 & 96 \\
Hyponatremia (N of cases) & 63 & 15 & 48 \\
Creatinine (mean $\pm \mathrm{SD})$ & $133 \pm 53$ & $154 \pm 74$ & $128 \pm 47$ \\
Systolic blood pressure (mean $\pm \mathrm{SD})$ & $108 \pm 17$ & $102 \pm 16$ & $109 \pm 17$ \\
Anemia $<$ Hb 100 g/l $(\mathrm{N}$ of cases) & 7 & 3 & 4 \\
Haemoglobin $(\mathrm{g} / \mathrm{L}$; mean $\pm \mathrm{SD})$ & $133 \pm 24$ & $127 \pm 28$ & $134 \pm 23$ \\
Ascites $(\mathrm{N}$ of cases) & 41 & 7 & 34 \\
Paracentesis $(\mathrm{N}$ of cases) & 27 & 6 & 21 \\
SBP $(\mathrm{N}$ of cases) & 11 & 4 & 7 \\
\hline
\end{tabular}

$\mathrm{SBP}$ - spontaneous bacterial peritonitis

Tab. 3. Triggering factors for acute right heart failure.

\begin{tabular}{lc}
\hline Triggering factor & $\mathrm{n}(\%$ of total) \\
\hline infections & $21(17.9 \%)$ \\
supraventricular arrhythmia & $10(8.5 \%)$ \\
anaemia & $7(5.9 \%)$ \\
activation of systemic lupus erythematosus & $1(0.9 \%)$ \\
non-compliance & $1(0.9 \%)$ \\
unknown & $77(66 \%)$ \\
\hline
\end{tabular}

Tab. 4. Treatment of acute right heart failure in our patient group.

\begin{tabular}{lccc}
\hline & \multicolumn{3}{c}{ Hospitalizations } \\
\cline { 2 - 4 } (n) & $\begin{array}{c}\text { All } \\
(\mathrm{n}=117)\end{array}$ & $\begin{array}{c}\text { Cardiology ward } \\
(\mathrm{n}=96)\end{array}$ & $\begin{array}{c}\text { ICU } \\
(\mathrm{n}=21)\end{array}$ \\
\hline Mortality (n) & $15(13 \%)$ & $4(4 \%)$ & $11(52 \%)$ \\
bolus diuretics & $45(38 \%)$ & $43(45 \%)$ & $2(10 \%)$ \\
continuous diuretics & $72(62 \%)$ & $53(55 \%)$ & $19(91 \%)$ \\
dobutamine & $37(32 \%)$ & $19(20 \%)$ & $17(81 \%)$ \\
norepinephrine & $6(5 \%)$ & - & $6(29 \%)$ \\
levosimendan & $5(4 \%)$ & - & $5(24 \%)$ \\
CVVH & $5(4 \%)$ & - & $5(24 \%)$ \\
NIV & $10(9 \%)$ & - & $10(48 \%)$ \\
IMV & $1(1 \%)$ & - & $1(5 \%)$ \\
ECMO & $2(2 \%)$ & - & $2(10 \%)$ \\
\hline
\end{tabular}

CVVH - Continuous veno-venous hemofiltration, NIV - non- invasive ventilation, IMV - invasive mechanic ventilation, ECMO - Extracorporeal Membrane Oxygenotherapy

Tab. 5. Treatment comparison in patients who survived or died during ICU treatment. Percentages show the fractions of patients who died or survived when the particular treatment was administered.

\begin{tabular}{lccc}
\hline & $\begin{array}{c}\text { All } \\
(\mathrm{n}=21)\end{array}$ & $\begin{array}{c}\text { Survivors } \\
(\mathrm{n}=10)\end{array}$ & $\begin{array}{c}\text { Non-survivors } \\
(\mathrm{n}=11)\end{array}$ \\
\hline dobutamine & 18 & $7(39 \%)$ & $11(61 \%)$ \\
norepinephrine & 6 & $0(0 \%)$ & $6(100 \%)$ \\
levosimendan & 5 & $2(40 \%)$ & $3(60 \%)$ \\
NIV & 10 & $4(40 \%)$ & $6(60 \%)$ \\
IMV & 1 & $0(0 \%)$ & $1(100 \%)$ \\
CVVH & 5 & $2(40 \%)$ & $3(60 \%)$ \\
ECMO & 2 & $1(50 \%)$ & $1(50 \%)$ \\
\hline CVVH - Continuous veno-venous hemofiltration, NIV - non-invasive ventilation, \\
IMV - invasive mechanic ventilation, ECMO - Extracorporeal Membrane Oxy- \\
genotherapy
\end{tabular}

tistically significant. 23 patients had hypotension (systolic blood pressure $\leq 90 \mathrm{~mm} \mathrm{Hg}$ ) and 7 patients were anemic (HB below pod $100 \mathrm{~g} / \mathrm{L})$. All cases were in NYHA III/IV class on admission. Clinically manifest ascites was present in 41 cases $(35 \%)$. As- cites paracentesis was performed in 27 cases due to a suspected tension ascites. High incidence of spontaneous bacterial peritonitis (SBP) was detected in these patients $(40.7 \%)$. Among ICU patients treated with paracentesis, the incidence of SBP was as high as $66.7 \%$ (Tab. 2).

\section{Triggering factors for right heart failure decompensation}

Triggering factors of ARHF were identified in 40 cases (34\% of all hospitalizations) and included infection in 21 patients (17.9\%), supraventricular tachycardia in 10 patients $(8.5 \%)$, anemia in 7 patients (5.9\%). In 77 patients, however, the reason remained unknown (66\%). Identification of the cause of heart failure was however not associated with better prognosis (odds ratio 1.04, $\mathrm{p}=\mathrm{NS}$ ) (Tab. 3).

\section{Specific treatment of pulmonary hypertension}

All patients with $\mathrm{PAH}$ were treated with $\mathrm{PAH}$-specific vasodilation therapy - sildenafil (56\%), endothelin receptor antagonists ( $27 \%$ ), prostanoids $(41 \%)$, riociguat $(1.4 \%)$, combination therapy ( $40 \%$ ). 5 out of 11 patients with CTEPH had a history of PEA. 4 patients were using riociguat; in 3 cases, the reason was residual PH following PEA and in one case, it was due to inoperability.

Treatment during the hospitalization for right heart failure and in-hospital mortality

During hospitalization for ARHF, patients were treated according to the severity of their clinical status. All patients were treated with diuretics. In $38.5 \%$ of patients, bolus diuretic administration was sufficient but, in most cases $(61.5 \%)$, diuretics had to be administered continuously.

Inotropic therapy was initiated in the absence of diuresis and/or where evidence of low cardiac output or systemic hypotension was present. Dobutamine was used as a drug of choice in $31.6 \%$ of cases. Where the effect was insufficient, levosimendan (4.3\%) and norepinefrine $(5.1 \%)$ were added. Continuous veno-venous hemofiltration $(\mathrm{CVVH})$ for acute kidney failure was used in 5 cases ( $4.3 \%)$.

Non-invasive pulmonary ventilation (NIV) was initiated in 10 cases with significant respiratory failure due to ventilation-perfusion mismatch. Invasive mechanical ventilation (IMV) was used in 1 patient. Extracorporeal Membrane Oxygenotherapy (ECMO) was used in two cases. An overview of treatment methods and the comparison between ICU and CW are given in Table 4 .

Risk factors of in-hospital mortality were calculated based on all 117 admissions of 70 patients with ARHF.

In-hospital mortality was $12.8 \%$. On $\mathrm{CW}$, the mortality rate was very low (4\%); however, the mortality of patients admitted to ICU was $52.4 \%$. Therapy in ICU in survivors and non-survivors is described in Table 5.

Using univariate analysis, a higher risk of in-hospital mortality was associated with lower sodium level (OR 2.1 per $5 \mathrm{mmol} / \mathrm{L}$ decrease, CI 1.1-4.1, p = 0.03), severe forms of PAH - heritable PAH, CTD-PAH (OR 4.5, CI 1.6-12.7, p = 0.005) and PAH treated with combination specific therapy (OR 3.1, CI 1.0-9.5, p=0.047). Surprisingly, the association between mortality and increasing age revealed a reduced odds ratio (OR 0.66 per 10 years increase, CI $0.50-0.88, \mathrm{p}=0.004)$, meaning that in-hospital mortality was 


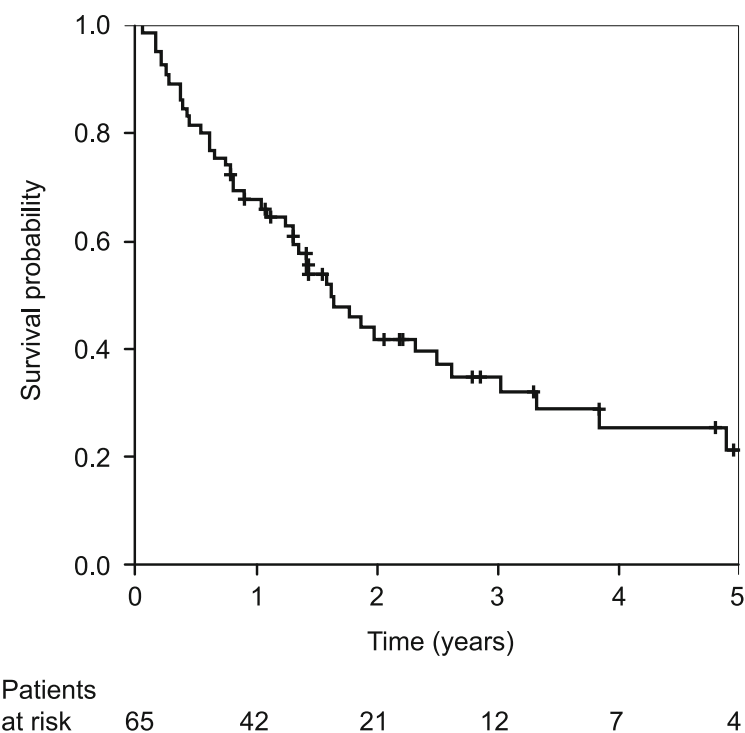

Fig. 2. Long-term survival of patients after the first admission for ARHF (only patients who were released from hospital care).

higher in younger patients. Sex, presence of ascites, time from diagnosis, serum levels of BUN, potassium, creatinine and identifiable triggering factor of ARHF did not predict in-hospital mortality.

\section{Long-term survival}

One-year survival after the first hospitalization (in patients who survived the first hospitalization) was $67.6 \%$ (95\% CI 57.1-80 $\%$ ); two years after the first hospitalization, it was $41.9 \%$ (95\% CI 30.8-56.9\%) (Fig. 2). In univariate analysis, the presence of ascites $(\mathrm{p}<0.02)$ and elevated BUN $(\mathrm{p}<0.009)$ on admission were associated with long-term mortality. A trend of higher mortality in patients with low blood pressure at the time of admission was also observed, however it was not statistically significant ( $p$ $=0.07$ ). Age, sex, identifiable cause of ARHF and haemodynamic profile at diagnosis were not significantly associated with longterm mortality after the first admission.

Age, presence of ascites, BUN level, sodium level, and blood pressure at the time of admission were included in the multivariate model. The choice of variables was guided by both clinical and statistical significance. In the multivariate analysis, only BUN was independently associated with the outcome.

\section{Discussion}

In our study, we focused on the description of risk factors and analysis of predictors of both hospital and long-term mortality. In addition, we also analysed the options of ARHF treatment. Neither the conclusions from the 6th World Symposium on Pulmonary Hypertension 2018 in Nice (1) nor the valid ESC Guidelines for the diagnosis and treatment of acute and chronic heart failure from 2016 (10) are dealing with the therapy of ARHF patients who are hemodynamically stable without clear clinical or laboratory indicators of low cardiac output or multiorgan failure and can there- fore be treated in standard cardiology wards. Besides targeting the underlying cause, a standard diuretic treatment is recommended (both bolus or continuous applications) as well as administration of spironolactone, oxygen therapy, low dose dobutamine (below $5 \mu \mathrm{g} / \mathrm{kg} / \mathrm{min}$ ) and symptomatic therapy. The in-hospital mortality of patients who required only this kind of treatment was relatively low (4\%). This result, however, cannot be reliably compared to previous studies on ARHF as none of those reported mortality separately for patients treated in cardiology ward $(2,3,11)$. The overall in-hospital mortality in our study was $12.8 \%$, which was caused by a high mortality of patients admitted to ICU (52.4\%). Similar results were reported by Campo et al (2) who reported 205 hospitalizations for ARHF in 90 patients, $16.1 \%$ of whom needed ICU treatment. The overall hospital mortality in their study was $8.8 \%$ and the ICU mortality was $48 \%$. In cases hospitalized at ICU who developed respiratory failure and invasive mechanic ventilation (IMV) was necessary $(n=9)$, the mortality was $100 \%$. Their work however did not specify whether or not non-invasive ventilation (NIV) was used in any patient (ventilation support was initiated in nine patients, however, the type of support was not reported).

In our patient group, the hospital mortality of patients treated at ICU who needed NIV due to respiratory failure was $60 \%$ (n $=10 ; 48 \%$ of ICU-treated patients). In one case, the ventilation support had to be changed to IMV, the patient however died. The indications for initiation of IMV and NIV are of course not interchangeable; however, where the patient's condition and ICU equipment allow a choice between IMV and NIV, it appears reasonable to begin with NIV and only if this approach fails, IMV and/or ECMO should be considered.

In patients hospitalized at ICU due to low cardiac output (LCO) accompanying right ventricular failure, inotropic support is administered, initially typically dobutamine. At doses $<5 \mu \mathrm{g} / \mathrm{kg} /$ min, dobutamine increases cardiac output and reduces PVR (12). Higher doses however often cause unwanted sinus tachycardia or development of arrhythmia and doses over $10 \mu \mathrm{g} / \mathrm{kg} / \mathrm{min}$ may lead to progression of systemic hypotension (12) as well as increased myocardial oxygen consumption.

If LCO symptoms and/or multiorgan failure persist, administration of levosimendan may be beneficial. Systemic hypotension often represents a limitation. When the systolic blood pressure is over $90 \mathrm{mmHg}$, it may be more beneficial to administer levosimendan than norepinephrine type vasopressors for increasing the cardiac output. In our study, 5 patients were treated in this way and the mortality was relatively high, i.e., $60 \%$. However, where the patients' conditions required administration of norepinephrine, the mortality was $100 \%$ (which is most likely associated with the generally extremely serious condition of those patients). Still, it is necessary to point out that norepinephrine acts as a strong vasoconstrictor both in the systemic and pulmonary vascular bed while levosimendan acts as a vasodilation agent in the pulmonary vascular bed. Hence, it is possible that administration of norepinephrine can possibly even worsen the prognosis of such critically ill patients and that levosimendan might indeed be the better choice.

As far as risk factors are concerned, our results are in accordance with those of previous works by Campo (2), Sztrymf (13) 
and Kurzyna (11). Lower sodium levels on admission and severe forms of PAH (HPAH, CTD-PAH) were statistically significant predictors for in-hospital mortality.

An interesting and previously unpublished observation was the relationship between in-hospital mortality and patient's age. Curiously enough, the in-hospital mortality in younger patients is higher than in older patients, which is probably associated with a higher risk profile of $\mathrm{PH}$ (PAH, especially CTD-PAH and heritable $\mathrm{PAH}$, necessity of PAH combination treatment) in younger patients.

Long-term mortality (Fig. 2) after the first hospitalization due to ARHF (32.4\% after 12 months and 58.1\% after 24 months) very well corresponds with the results published by Campo (2) (35\% at month 12 , mortality at month 24 is not reported) despite the fact that the spectrum of patients differs (in Campo's paper, there was a high representation of patients with CTD-PAH while no patients with CTEPH were included).

The presence of ascites turned out to be a predictor of longterm mortality, which can be explained by the well-known fact that ascites is a sign of very advanced disease. Besides, a high percentage of SBP found in patients with ascites that is correctly treated with antibiotics during the hospitalization but not in a long term after the release from the hospital (which is recommended when the aetiology of ascites is cirrhosis) (14) may be the reason for such worsening of the long-term outlook.

Limitations of this study include especially relatively small number of patients, which prevents us from providing a proper statistical analysis and only allows a description of the results, a relatively high heterogeneity of the patient group (various forms of PAH, CTEPH) and an absence of data from the right heart catheterization at the time of hospitalization.

\section{Conclusions}

In-hospital mortality of patients with pulmonary hypertension and ARHF is high, and significantly higher when patients need treatment in intensive care units. Higher risk of in-hospital mortality was associated with younger age, lower sodium levels, severe forms of PAH (heritable PAH, CTD-PAH) and need of PAH combination treatment. The presence of ascites and BUN levels were the most important predictors of long-term mortality. This mortality could be also influenced by high prevalence of SBP in patients with ascites, which was treated with antibiotics only during the in-hospital stay, but not in the long term as is recommended in patients with cirrhosis ascites.

\section{Learning points}

- Mortality in patients with PH and ARHF remains very high.

- A higher risk of in-hospital mortality of patients with ARHF and pulmonary hypertension was found in patients with younger age, lower sodium levels, severe forms of PAH (heritable PAH, CTD-PAH) and patients requiring PAH combination treatment.

- The presence of ascites was not associated with a higher hospital mortality but was a predictor of long-term mortality.

- If ventilation is needed in these patients, it appears reasonable to begin with non-invasive ventilation and only where this approach fails, IMV and/or ECMO should be considered.

- Identification of mortality risk factors could be used as basis of risk-adapted therapy.

\section{References}

1. Hoeper MM, Benza RL, Corris P, de Perrot M, Fadel E, Keogh AM et al. Intensive care, right ventricular support and lung transplantation in patients with pulmonary hypertension. Eur Resp J 2019; 53 (1): 1801906.

2. Campo A, Mathai SC, Le Pavec J, Zaiman AL, Hummers LK, Boyce D et al. Outcomes of hospitalisation for right heart failure in pulmonary arterial hypertension. Eur Respir J 2011; 38 (2): 359-367.

3. Haddad F, Peterson T, Fuh E, Kudelko KT, de Jesus Perez V, Skhiri $\mathbf{M}$ et al. Characteristics and outcome after hospitalization for acute right heart failure in patients with pulmonary arterial hypertension. Circulat Heart Fail 2011; 4 (6): 692-699.

4. Wang S, Yu M, Zheng X, Dong S. A Bayesian network meta-analysis on the efficacy and safety of eighteen targeted drugs or drug combinations for pulmonary arterial hypertension. Drug Deliv 2018; 25 (1): 1898-1909.

5. Zheng Y-G, Ma H, Chen L, Jiang X-M, Zhou L, Lin S et al. Efficacy and safety of oral targeted therapies in pulmonary arterial hypertension: a meta-analysis of randomized clinical trials. Pulmon Circulat 2018; 8 (4): 2045894018798183.

6. Kaestner M, Schranz D, Warnecke G, Apitz C, Hansmann G, Miera O. Pulmonary hypertension in the intensive care unit. Expert consensus statement on the diagnosis and treatment of paediatric pulmonary hypertension. The European Paediatric Pulmonary Vascular Disease Network, endorsed by ISHLT and DGPK. Heart 2016; 102 (2): 2015-307774.

7. Hoeper MM, Granton J. Intensive care unit management of patients with severe pulmonary hypertension and right heart failure. Am J Respir Crit Care Med 2011; 184 (10): 1114-1124.

8. Frost A, Badesch D, Gibbs JSR, Gopalan D, Khanna D, Manes A et al. Diagnosis of pulmonary hypertension. Eur Resp J 2019; 53 (1): 1801904.

9. Kim NH, Delcroix M, Jais X, Madani MM, Matsubara H, Mayer E et al. Chronic thromboembolic pulmonary hypertension. Eur Resp J 2019; 53 (1): 1801915.

10. Ponikowski P, Voors AA, Anker SD, Bueno H, Cleland JGF, Coats AJS et al. 2016 ESC Guidelines for the diagnosis and treatment of acute and chronic heart failure: The Task Force for the diagnosis and treatment of acute and chronic heart failure of the European Society of Cardiology (ESC) Developed with the special contribution of the Heart Failure Association (HFA) of the ESC. Eur Heart J 2016; 37 (27): 2129-2200.

11. Kurzyna M, Zylkowska J, Fijalkowska A, Florczyk M, Wieteska M, Kacprzak A et al. Characteristics and prognosis of patients with decompensated right ventricular failure during the course of pulmonary hypertension. Kardiol Pol 2008; 66 (10): 1033-1039.

12. Price LC, Wort SJ, Finney SJ, Marino PS, Brett SJ. Pulmonary vascular and right ventricular dysfunction in adult critical care: current and emerging options for management: a systematic literature review. Crit Care 2010; 14 (5): 21.

13. Sztrymf B, Souza R, Bertoletti L, Jais X, Sitbon O, Price LC et al. Prognostic factors of acute heart failure in patients with pulmonary arterial hypertension. Eur Respir J 2010; 35 (6): 1286-1293.

14. EASL Clinical Practice Guidelines for the management of patients with decompensated cirrhosis. J Hepatol 2018; 69 (2): 406-460.

Received July 15, 2019. Accepted October 3, 2019. 